EPJ Web of Conferences 38, 03003 (2012)

DOI: $10.1051 /$ epjconf/20123803003

(C) Owned by the authors, published by EDP Sciences, 2012

\title{
Physics of unstable nuclei: from structure to sequential decays
}

\author{
Alexander Volya ${ }^{\mathrm{a}}$ \\ Department of Physics, Florida State University, Tallahassee, Florida 32306-4350, USA.
}

\begin{abstract}
In this presentation we discuss features of nuclear structure and properties of nuclear decays that result from coupling to the reaction states with one and two nucleons in the continuum. Physics of the one-body decays is reviewed, and perturbations due to the virtual excitations into the continuum of reaction states are examined. The two-body decays are explored using the sequential decay mechanism. The two-neutron s-wave breakups are of particular interest, here the two-body nature of the process appears to suppress the decay rate as compared to the kinematically equivalent one-body s-wave decay. The effective phase space scaling and the resulting from it decay width as a function of the available energy are examined for identical and non-identical particles assuming different values of the scattering length.
\end{abstract}

\section{Introduction}

Experimental observations of multi-nucleon decays, such as the ones reported in Refs. [1-4], challenge our theoretical understanding of the nuclear dynamics on the verge of stability. Tying together the nuclear many-body structure and physics of decay is not an easy task. Selected recent efforts in this direction can be found in Refs. [5-8]. In this contribution we discuss some generic aspects of particle decay, interplay between structure and reactions, and features of the three-body breakup.

\section{One-body decay}

\subsection{Single particle decay amplitude}

We assume that a one-body decay $I_{1} \rightarrow I_{2}$, illustrated in figure 1 , proceeds due to an interaction component of the Hamiltonian $H_{Q P_{1}}$. In this process a resonant state $I_{1}$ emits a particle with energy $\epsilon$, and the system transitions into a final state $I_{2}$ of the daughter nucleus. In the Feshbach projection formalism used in Refs. [6,9] the component $H_{Q P_{1}}$ is the one that connects the intrinsic space $Q$ with the one-body continuum space $P_{1}$. The external space $P=P_{1}+P_{2} \ldots$ where the subscript denotes the number of particles in reaction continuum. The decay amplitude is defined as

$$
A_{1,2}(\epsilon)=\left\langle I_{2}, \epsilon\left|H_{Q P_{1}}\right| I_{1}\right\rangle .
$$

\footnotetext{
a e-mail: avolya@fsu.edu
}

1

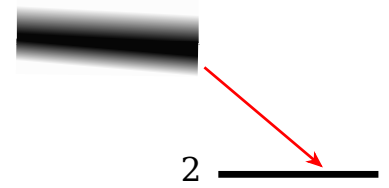

Fig. 1. Illustration of the one-body decay process $I_{1} \rightarrow I_{2}$.
The $I_{1} \stackrel{\epsilon}{\rightarrow} I_{2}$ partial decay width, corresponding to this amplitude, is given by the Fermi Golden Rule

$$
d \Gamma_{1,2}(\epsilon)=2 \pi\left|A_{1,2}(\epsilon)\right|^{2} \delta\left(E_{1}-E_{2}-\epsilon\right) d \epsilon .
$$

The same result follows from the Feshbach projection formalism, see Refs. [6,9]. The total width is a function of the available energy $\epsilon=E_{1}-E_{2}$,

$$
\Gamma_{1,2}(\epsilon)=2 \pi\left|A_{1,2}(\epsilon)\right|^{2} .
$$

The one-body continuum states $\left|I_{2}, \epsilon\right\rangle$ in the space $P_{1}$ are energy-delta-function normalized; therefore the amplitudes are normalized to include the density of states. Direct evaluation of the overlap amplitude in Eq. (3) is a common method of evaluating the single particle decay width [10]. If the one-body Hamiltonian $H_{Q P_{1}}$ is given by a spherically symmetric potential $V(r)$ then the amplitude in Eq. (1) is

$$
A_{1,2}(\epsilon)=\sqrt{\frac{2 \mu}{\pi \hbar^{2} k}} \int_{0}^{\infty} d r u_{I_{1}}(r) V(r) F_{I_{2}}(k r) .
$$

Here $k=\sqrt{2 \mu \epsilon}$; the initial state $I_{1}$ is described by a singleparticle radial wave function $u_{I_{1}}(r)$; the final state in the continuum is given by the Coulomb (Bessel) function $F_{I_{2}}(k r)$; and the coefficient in front of the integral reflects the energydelta-function normalization of states in the continuum. At low energies the energy-dependence of the amplitude is determined by the asymptotic of the function $F_{I_{2}}(k r)$ as $k r \rightarrow 0$. In this limit the dependence of the decay width on energy is commonly expressed using the phenomenological R-matrix formalism [11] via scaling $\chi$ and the channel radius parameter $R$ :

$$
\Gamma(\epsilon) \simeq 2 \chi \gamma_{w}(R) \mathcal{P}(k R)
$$

where

$$
\mathcal{P}(k R)=\frac{k R}{F^{2}(k R)+G^{2}(k R)} \quad \text { and } \quad \gamma_{w}(R)=\frac{3 \hbar^{2}}{2 \mu R^{2}} .
$$

Here $G$ is the irregular Coulomb (Bessel) function. In this work we discuss neutral particles, in which case $\mathcal{P}_{0}(x)=x$ 


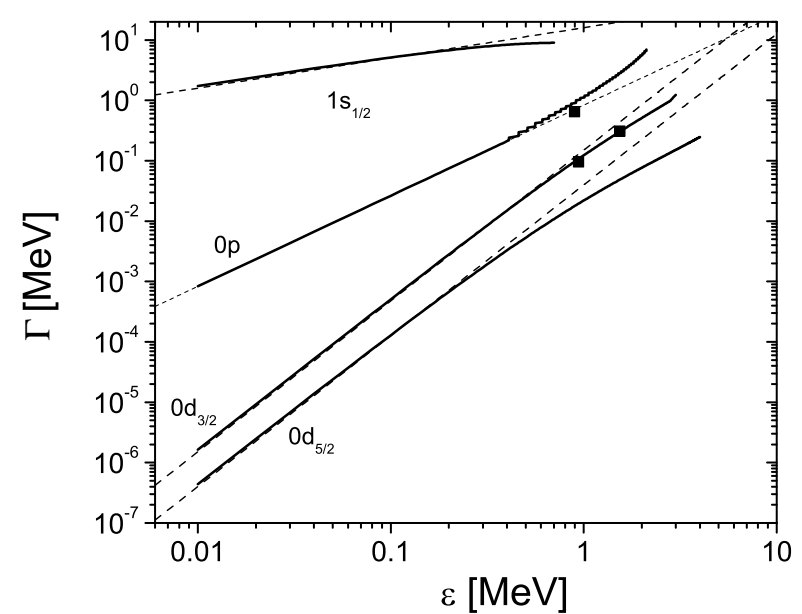

Fig. 2. Energy dependence of the single-particle decay widths for neutral particles. Thin straight lines show the power-law scaling $\Gamma \propto \epsilon^{\beta}$ where $\beta=l+1 / 2$, as follows from Eq. (5) in the $\epsilon \rightarrow 0$ limit; solid lines represent calculations with Eq. (4). Experimental points are marked for ${ }^{5} \mathrm{He}(\epsilon=0.895 \mathrm{MeV}, \Gamma=648 \mathrm{keV}),{ }^{17} \mathrm{O}$ $(\epsilon=0.941 \mathrm{MeV}, \Gamma=98 \mathrm{keV})$, and ${ }^{19} \mathrm{O}(\epsilon=1.540 \mathrm{MeV}, \Gamma=310$ $\mathrm{keV})$, see Ref. [6].

and $\mathcal{P}_{1}(x)=x^{3} /\left(1+x^{2}\right)$, in general $\mathcal{P}_{l}(x) \propto x^{2 l+1}$ when $x \ll l+1 / 2$. The dependence of the single-particle decay width on energy is shown in figure 2 . At high energies the amplitude goes to zero and the treatment is analogous to Born approximation.

\subsection{Interaction with one-body continuum}

The coupling to the continuum amounts to an additional effective interaction Hamiltonian $[6,9]$ in the internal space $Q$

$$
H^{\prime}(\epsilon)=\int_{0}^{\infty} d \epsilon^{\prime} \frac{\left|A\left(\epsilon^{\prime}\right)\right|^{2}}{\epsilon-\epsilon^{\prime}+i 0}
$$

Here, we assume an on-shell form which emerges when the internal and continuum spaces are orthogonal; the general expression and its derivation can be found in Ref. [6,9]. In the single channel example for brevity of notations the subscripts are omitted, $A_{1,2} \equiv A$, and all energies are measured form the channel's threshold $E=\epsilon$. The interaction in Eq. (7) is analogous to the second order energy perturbation due to excitations into the continuum. The Feshbach projection formalism, however, is exact; the exact eigenvalues in the full space are found from an energy dependent Hamiltonian $\mathcal{H}(E)=H_{Q Q}+H^{\prime}(E)$ using the nonlinear version of Schroedinger's equation $\mathcal{H}(E)|I\rangle=E|I\rangle$. In general $H^{\prime}$ and $\mathcal{H}$ are non-Hermitian. If for some decay channel $\epsilon>0$ then the integration region in Eq. (7) contains a pole and the integral can be separated into the Hermitian principal value part $\Delta(\epsilon)$ (self-energy) and the remaining non-Hermitian term $\Gamma(\epsilon)$ (the decay width):

$$
H^{\prime}(\epsilon)=\Delta(\epsilon)-\frac{i}{2} \Gamma(\epsilon)
$$

where

$$
\Delta(\epsilon)=\int d \epsilon^{\prime} \frac{\left|A\left(\epsilon^{\prime}\right)\right|^{2}}{\epsilon-\epsilon^{\prime}+i 0}, \quad \Gamma(\epsilon)=2 \pi|A(\epsilon)|^{2} .
$$

The real and imaginary parts of $H^{\prime}$ are shown in figure 3 for ${ }^{17} \mathrm{O}$. In this example we assume an intrinsic state in the space $Q$ to have a harmonic oscillator wave function, the oscillator frequency being $\omega=41 A^{-1 / 3} \mathrm{MeV}$. The coupling to continuum $H_{Q P_{1}}$ in Eq. (4) is modeled by a WoodsSaxon potential. It was found that for both s- and d-waves the amplitude at low energies is well parameterized by Eq. (5) with $\chi=0.256$ and $R=4.81 \mathrm{fm}$.

The role of the non-Hermitian part $\Gamma$ in the nuclear many-body problem is discussed in Refs. $[6,9]$. This term has a factorized form, which maintains the unitarity of the scattering matrix. The factorized form leads to a dynamical phase transition [12], also known as superradiance [13]. The Hermitian correction from virtual excitations into the continuum, $\Delta(\epsilon)$, modifies positions of bound and resonant states near decay thresholds. Sharp changes in spectroscopic observables near thresholds have been studied in Ref. [14]. The strongest, cusp-like effect is seen in figure 3 for the s-wave, $\Delta_{0}(0)=-1.2 \mathrm{MeV}$. For other angular momentum channels the magnitude of $\Delta(\epsilon)$ is smaller and its energy behavior is more continuous. The importance of the self-energy term in near-dripline nuclei is discussed in Ref. [9]. The subject, however, is far from being settled. First, most successful effective shell model interactions are adjusted to experimental observables and therefore include the self-energy in some averaged form. Second, the phenomenological shell model implies some mean-field singleparticle basis with wave functions that are more realistic than those of the harmonic oscillator examined figure 3, therefore the resulting self-energy corrections are small. Moreover, the stationary bound states of a given mean field potential, such as solutions of the Woods-Saxon potential, are eigenstates which are not subject to any additional perturbation due to the one-body continuum.

\section{Two-body decay}

\subsection{Sequential decay}

In this section we continue the discussion in Ref. [6] focusing on selected aspects of the two-body decay, related studies can be found in Ref. [15]. Here a system is coupled to states involving more than one particle in the continuum. The two-body decay represents a transition from $Q$ to $P_{2}$. This can be generated by a direct three-body interaction Hamiltonian $\mathrm{H}_{\mathrm{QP}_{2}}$ or by a second order sequential process involving $H_{Q P_{1}}$. We examine the second case, referred to as the sequential decay $I_{1} \stackrel{\epsilon_{1}}{\rightarrow} I_{2} \stackrel{\epsilon_{2}}{\rightarrow} I_{3}$, see illustration in figure 4. A somewhat analogous process takes place in the two-photon decay from the 2 s state in the Hydrogen atom.

The amplitude for the two-body decay $I_{1} \stackrel{\epsilon_{1}}{\rightarrow} I_{2} \stackrel{\epsilon_{2}}{\rightarrow} I_{3}$ is

$$
A_{1,2,3}\left(\epsilon_{1}, \epsilon_{2}\right)=\frac{A_{1}\left(\epsilon_{1}\right) A_{2}\left(\epsilon_{2}\right)}{\epsilon_{2}-\left(E_{2}-\frac{i}{2} \Gamma_{2,3}\left(\epsilon_{2}\right)\right)} .
$$

Here we present an on-shell form $E_{1}=\epsilon_{1}+\epsilon_{2}+E_{3}$; we also assume that the width of the intermediate state is fully determined by the single decay channel $I_{2} \stackrel{\epsilon_{2}}{\rightarrow} I_{3}$; and, finally, we set the energy scale so that $E_{3}=0$. The Fermi Golden Rule gives the partial decay width distribution for the sequential process as

$$
\frac{d \Gamma(E)}{d \epsilon_{1} d \epsilon_{2}}=2 \pi \delta\left(E-\epsilon_{1}-\epsilon_{2}\right)\left|A_{T}\left(\epsilon_{1}, \epsilon_{2}\right)\right|^{2} .
$$




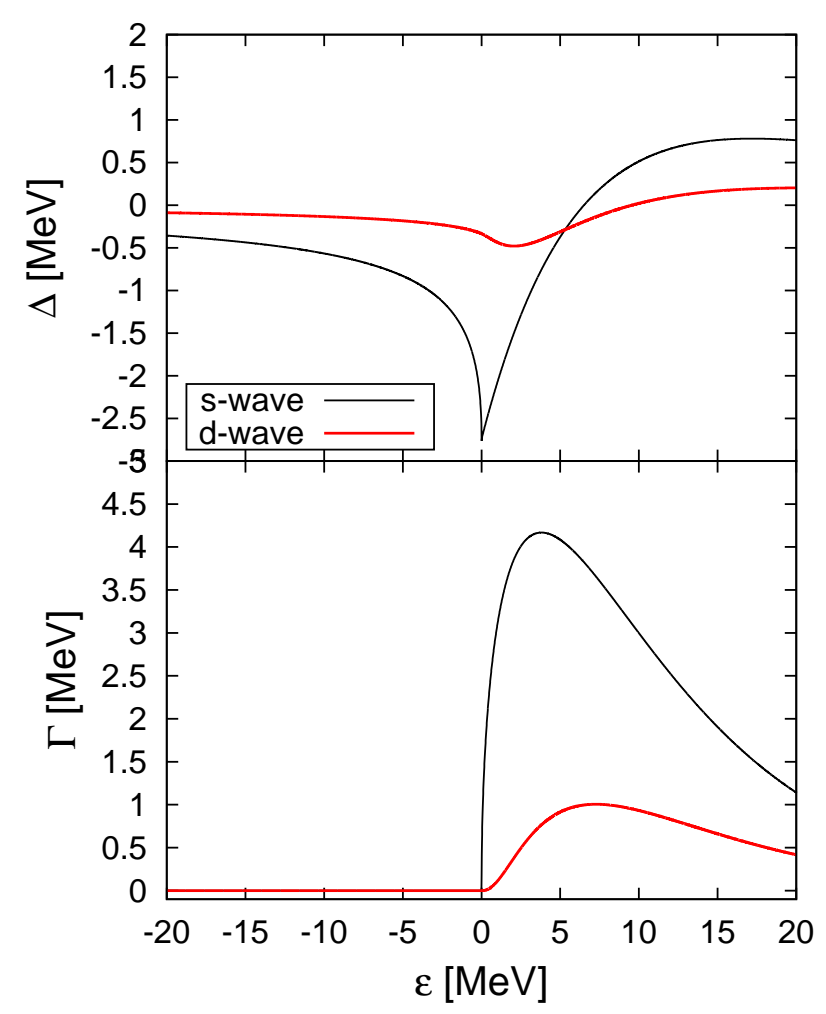

Fig. 3. Example of corrections due to the one-body continuum in ${ }^{17} \mathrm{O}$. Self-energy $\Delta(\epsilon)$ and width $\Gamma(\epsilon)$ are shown as functions of energy $\epsilon$ measured from the threshold.

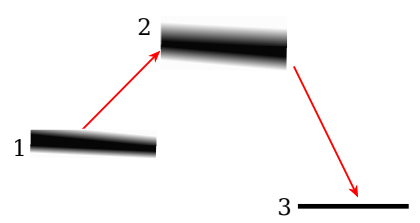

Fig. 4. Illustration of the sequential decay process $I_{1} \stackrel{\epsilon_{1}}{\rightarrow} I_{2} \stackrel{\epsilon_{2}}{\rightarrow} I_{3}$.

The total amplitude $A_{T}$ in Eq. (9) must include amplitudes for all intermediate states, and it should be (anti) symmetrized for identical particles.

When $E_{2}>E_{1}$, this case is illustrated in figure 4, the intermediate state is higher in energy which creates an energy barrier for the decay. The traditional sequential process occurs when $E_{2}<E_{1}$ and the direct one-body decay channel is open leading to an emission of the first particle with energy $\epsilon_{1}=E_{1}-E_{2}$. In this case the amplitude (8) has a pole at this energy (at the pole $E_{2}=\epsilon_{2}$ ). If an intermediate state is narrow one can use the following approximation $\Gamma /|E-i \Gamma / 2|^{2} \approx 2 \pi \delta(E)$. This approximation leads to the classically expected result that the lifetime of the initial state is fully determined by the first step in the sequential decay $I_{1} \stackrel{\epsilon_{1}}{\rightarrow} I_{2}$, therefore $\Gamma_{1,2,3}=\Gamma_{1,2}$.

In figure 5 the integrated two-body sequential decay width is shown as a function of $E_{2}$ in the case when both particles are emitted in the $d$-wave. It is assumed that $E_{1}=$ $1 \mathrm{MeV}\left(E_{3}=0\right)$. While $E_{2}<E_{1}$ and the direct singleparticle decay channel is open $\Gamma_{1,2,3} \simeq \Gamma_{1,2}$. In figure 5 the one-body width $\Gamma_{1,2}$ (blue dashed line) coincides with $\Gamma_{1,2,3}$ (red solid line) in this limit. At energies $E_{2}>E_{1}$,

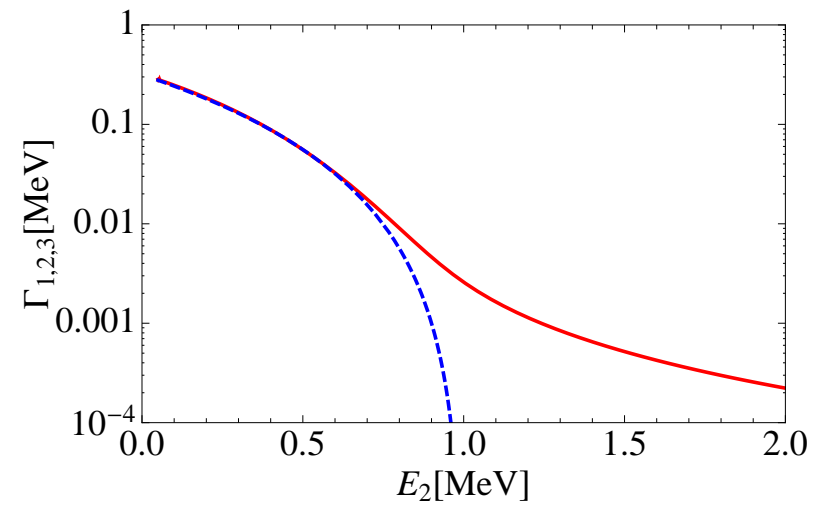

Fig. 5. Sequential $d$-wave two-nucleon decay width $\Gamma_{1,2,3}$ is shown as a function of the intermediate state's energy $E_{2}$ (red line). The initial state is at energy $E_{1}=1 \mathrm{MeV}$; the final state is at $E_{3}=0 \mathrm{MeV}$. The single-particle decay width is assumed to be $\Gamma_{1,2}(\epsilon)=\Gamma_{2,3}(\epsilon)=0.3158 \epsilon^{5 / 2}(\epsilon$ and $\Gamma$ are in units of $\mathrm{MeV})$. The $\Gamma_{1,2}\left(E_{1}-E_{2}\right)$ is shown with dashed-blue line.

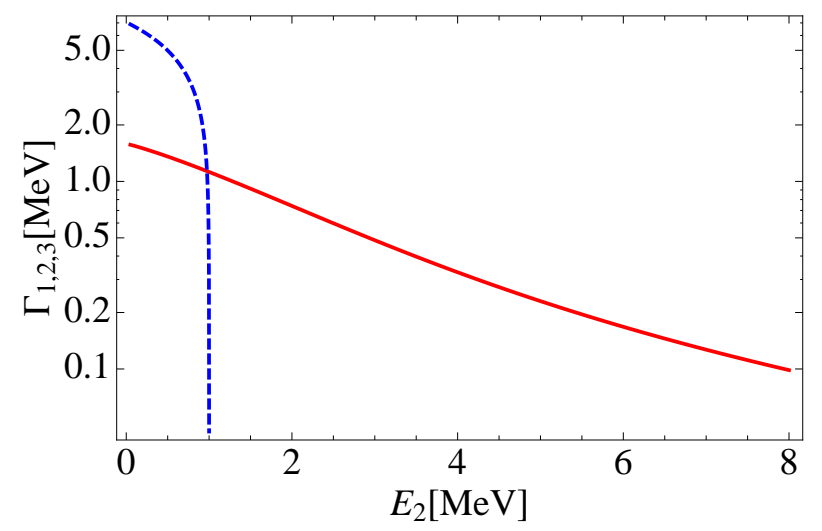

Fig. 6. Same as in Fig. 5 but assuming an s-wave process. The single-particle decay width is parameterized as $\Gamma_{1,2}(\epsilon)=$ $\Gamma_{2,3}(\epsilon)=7.0 \sqrt{\epsilon}(\epsilon$ and $\Gamma$ are in units of $\mathrm{MeV})$.

the sequential decay proceeds via a virtual resonant state, while the direct one-body decay is no longer possible.

In the case when both neutrons are emitted in the $s$ wave, figure 6 , the narrow width approximation is no longer valid. It may be counterintuitive, but, as seen from figure 6 , the condition $\Gamma_{1,2,3}=\Gamma_{1,2}$, expected classically for a sequential process, is not fulfilled. Due to the extremely short-lived nature of an intermediate state it is appropriate to interpret the process as a three-body breakup rather than as sequential. It is interesting that the lifetime of the initial state $I_{1}$ becomes longer if its daughter product, the state $I_{2}$, is unstable to further s-wave decay $I_{2} \rightarrow I_{3}$. For $E_{2}<1 \mathrm{MeV}$ in figure 6 the sequential decay width $\Gamma_{1,2,3}$ (in solid red) is smaller than $\Gamma_{1,2}$ (in dashed-blue). Narrow three-body s-wave states have also been discussed in Ref. [16].

\subsection{Low energy s-wave decay}

In this section we concentrate on the s-wave three-body breakup. We assume the one-body decay width to be given by Eq. 5 with $\chi=2 / 3$, see Ref. [17], and $\Gamma_{1}(\epsilon)=\frac{2 \hbar^{2}}{\mu_{1} R_{1}} k$, where $R_{1}$ is the channel radius parameter for decay from the resonance $I_{1}$. In the s-wave process the decay width, 
which is proportional to $\sqrt{\epsilon}$, is the leading term in the denominator of Eq. (8), thus the form of the scattering amplitude in the effective range expansion is recovered [18],

$$
\epsilon_{2}-\left(E_{2}-\frac{i}{2} \Gamma_{2}\left(\epsilon_{2}\right)\right) \approx \frac{\hbar^{2}}{\mu_{2} R_{2}}\left(\frac{1}{a_{2}}+i k_{2}\right) .
$$

This transfers the sequential picture of decay that takes place through a resonant state into a three-body breakup where an intermediate structure is described by the lowenergy s-wave behavior. This behavior is controlled by the scattering length $a_{2}$ or equivalently with the energy parameter $\varepsilon_{2}$ :

$$
a_{2} \equiv \frac{\Gamma_{2}(\epsilon)}{2 k E_{2}}=\frac{\hbar^{2}}{\mu_{2} R_{2} E_{2}}, \quad \varepsilon_{2} \equiv \frac{\hbar^{2}}{2 m_{2} a_{2}^{2}} .
$$

In the following using Eq. (10) we substitute $E_{2}$ with the virtual state energy $\varepsilon_{2}$ which, being tied to the scattering length, is a universal property of the s-wave scattering at low energies. As a result of this substitution for distinct particles, where the total amplitude is given by Eq.(8), we obtain

$$
\frac{d \Gamma_{1,2,3}\left(\epsilon_{1} \epsilon_{2}\right)}{d \epsilon^{\prime}}=\frac{2 \lambda}{\pi} \frac{\sqrt{\left(E_{1}-\epsilon_{2}\right) \epsilon_{2}}}{\varepsilon_{2}+\epsilon_{2}} .
$$

Here

$$
\lambda=\frac{R_{2}}{R_{1}} \sqrt{\frac{\mu_{2}}{\mu_{1}}}
$$

is a dimensionless parameter that is expected to be close to unity. The integrated decay width as a function of the resonance energy $E_{1}$ is

$$
\Gamma_{1,2,3}\left(E_{1}\right)=\lambda\left[2 \varepsilon_{2}+E_{1}-2 \sqrt{\varepsilon_{2}\left(E_{1}+\varepsilon_{2}\right)}\right] .
$$

The phase space volume is an important factor in a reaction rate; at low energies it leads to a power law $\Gamma(E) \propto$ $E^{\beta}$, see also Refs. $[6,8]$. Let us examine the value of the scaling parameter $\beta$ for different processes. The one-body phase space leads to $\beta=1 / 2$ for the $s$-wave (symmetric) decay. The three-body phase space scales with energy with $\beta=2$ since $\int d^{3} k_{1} d^{3} k_{2} \delta\left(E-\epsilon_{1}-\epsilon_{2}\right) \propto E^{2}$; and indeed at very low energies when $E_{1} \ll \varepsilon_{2}$ we find from Eq. (13)

$$
\Gamma_{1,2,3}\left(E_{1}\right) \simeq \lambda \frac{E_{1}^{2}}{4 \varepsilon_{2}} .
$$

In the other limit $E_{1} \gg \varepsilon_{2}$, at higher energies or when the scattering length is large compared to the particle's wave length at the decay energy,

$$
\Gamma_{1,2,3}\left(E_{1}\right) \simeq \lambda E_{1},
$$

and $\beta=1$. This confirms that the decay is different from sequential because the scaling law is not that of $\beta=1 / 2$.

For identical particles the total amplitude is $A_{T}^{ \pm}\left(\epsilon_{1}, \epsilon_{2}\right)=$ $\left[A_{1,2,3}\left(\epsilon_{1}, \epsilon_{2}\right) \pm A_{1,2,3}\left(\epsilon_{2}, \epsilon_{1}\right)\right] / \sqrt{2}$. The limit where $E_{1} \gg$ $\varepsilon_{2}$ is similar and corresponds to $\beta=1$,

$$
\Gamma_{1,2,3}^{ \pm}\left(E_{1}\right) \simeq \frac{\pi \pm 2}{\pi} \lambda E_{1} .
$$

At low energies, $E_{1} \ll \varepsilon_{2}$, the symmetric amplitude again leads to $\beta=2$,

$$
\Gamma_{1,2,3}^{+}\left(E_{1}\right) \simeq \lambda \frac{E_{1}^{2}}{2 \varepsilon_{2}} ;
$$

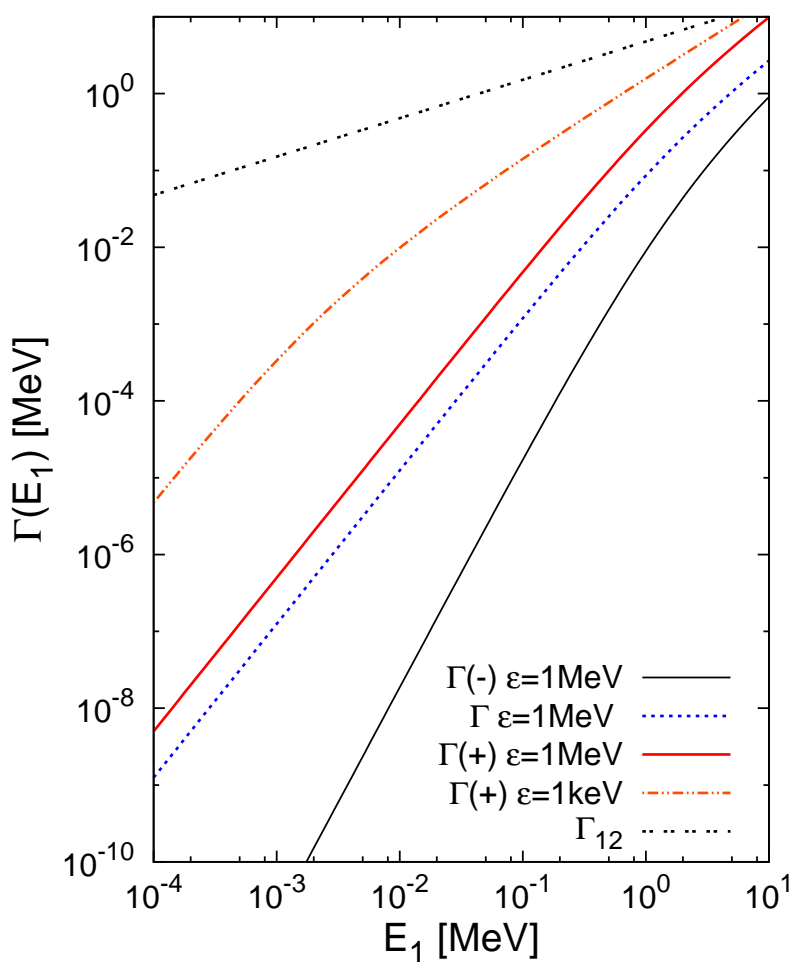

Fig. 7. $\Gamma_{1,2,3}^{ \pm}\left(E_{1}\right)$ (labeled as $\Gamma(+)$ and $\Gamma(-)$ ) and $\Gamma_{1,2,3}\left(E_{1}\right)$ for distinguishable particles (labeled as $\Gamma$ ) are shown as functions of the total decay energy $E_{1}$. The values of $\varepsilon_{2}$ are indicated. The one-body decay width $\Gamma_{1,2}\left(E_{1}\right)$ as a function of energy is also shown.

however, the destructive interference of terms in the antisymmetric amplitude results in $\beta=3$ in $E_{1} \ll \varepsilon_{2}$ limit,

$$
\Gamma_{1,2,3}^{-}\left(E_{1}\right) \simeq \frac{3 \pi-8}{24 \pi} \lambda \frac{E_{1}^{3}}{\varepsilon_{2}^{2}} .
$$

In figure 7 the quantity $\Gamma_{1,2,3}\left(E_{1}\right)$ is shown as a function of $E_{1}$ for these cases. The one-body $\Gamma_{12}\left(E_{1}\right)$ is also included to highlight a relatively long-lived nature of configurations involving two s-wave neutrons. The finding appears to support ideas in Ref. [16]. It is remarkable that the predicted swave two-body decay width in the experimentally-relevant region of energy appears to follow a simple law $\Gamma \simeq E$. The changes in the phase-space energy scaling considered in this work emerge due to quantum evolution via an intermediate-state; similar discussions can be found in Refs. [1, $16,19,20]$.

This work is supported by the U.S. DOE grant DEFG02-92ER40750.

\section{References}

1. A. Spyrou, Z. Kohley, T. Baumann, D. Bazin, B. A. Brown, G. Christian, P.A. DeYoung, J.E. Finck, N. Frank, E. Lunderberg et al., Phys. Rev. Lett. 108, 102501 (2012)

2. C. R. Hoffman, T. Baumann, J. Brown, P.A. DeYoung, J.E. Finck, N. Frank, J.D. Hinnefeld, S. Mosby, W.A. Peters, W.F. Rogers et al., Phys. Rev. C 83, 031303 (2011) 
3. E. Lunderberg, P.A. DeYoung, Z. Kohley, H. Attanayake, T. Baumann, D. Bazin, G. Christian, D. Divaratne, S.M. Grimes, A. Haagsma et al., Phys. Rev. Lett. 108, 142503 (2012)

4. H.T. Johansson, Y. Aksyutina, T. Aumann, K. Boretzky, M.J.G. Borge, A. Chatillon, L.V. Chulkov, D. Cortina-Gil, U.D. Pramanik, H. Emling et al., Nucl. Phys. A847 (2010)

5. N. Michel, W. Nazarewicz, M. Ploszajczak, and J. Okolowicz, Phys. Rev. C 67, 054311 (2003)

6. A. Volya and V. Zelevinsky, Phys. Rev. C 74, 064314 (2006)

7. T. Myo, K. Kato, and K. Ikeda, Phys. Rev. C 76, 054309 (2007)

8. L.V. Grigorenko, I.G. Mukha, C. Scheidenberger, and M.V. Zhukov, Phys. Rev. C 84, 021303 (2011)

9. A. Volya, Phys. Rev. C 79, 044308 (2009)

10. S. Åberg, P.B. Semmes, and W. Nazarewics, Phys. Rev. C 56, 1762 (1997)

11. D. Baye and P. Descouvemont, Rep. Prog. Phys. 73, 036301 (2010)

12. I. Rotter, J. Phys. A 42, 153001 (2009)

13. N. Auerbach and V. Zelevinsky, Rep. Prog. Phys. 74, $106301(2011)$

14. N. Michel, W. Nazarewicz, M. Ploszajczak, and T. Vertse, J. Phys. G 36, 013101 (2009)

15. J. Rotureau, J. Okolowicz, and M. Ploszajczak, Nucl. Phys. A767, 13 (2006)

16. L.V. Grigorenko and M.V. Zhukov, Phys. Rev. C 77, 034611 (2008)

17. A. Bohr and B.R. Mottelson, Nuclear Structure (World Scientific Publishing, 1998)

18. L.D. Landau and E.M. Lifshitz, Quantum mechanics. Non-relativistic theory. (Pergamon Press, New York, 1981)

19. Steven E. Koonin, Phys. Lett. B 70, 43 (1977)

20. R. Lednicky and V.L. Lyuboshits, Sov. J. Nucl. Phys. 35 (1982) 